Proc. of the 15th Int. Workshop on Slow Positron Beam Techniques and Applications, Prague, September 2-6, 2019

\title{
Development of Data-Analysis Software for Total-Reflection High-Energy Positron Diffraction (TRHEPD)
}

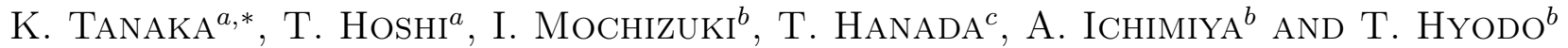 \\ ${ }^{a}$ Department of Applied Mathematics and Physics, Tottori University, \\ 4-101 Koyama-Minami, Tottori 680-8550, Japan \\ ${ }^{b}$ Institute of Materials Structure Science, High Energy Accelerator Research Organization, \\ 1-1 Oho, Tsukuba, Ibaraki 305-0801, Japan \\ ${ }^{c}$ Institute for Materials Research, Tohoku University, 2-1-1 Katahira, Aoba-ku, Sendai 980-8577, Japan
}

The present paper reports on the recent activity of the data analysis software development for total-reflection high-energy positron diffraction, a novel experimental technique for surface structure determination. Experiments using TRHEPD are being conducted intensively at the Slow Positron Facility, Institute of Materials Structure Science, High Energy Accelerator Research Organization, revealing surface structure of interest. The data analysis software provides a solution to the inverse problem in which the atomic positions of a surface structure are determined from the experimental diffraction data (rocking curve). The forward problem is solved by the numerical solution of the partial differential equation in the quantum scattering problem. A technical demonstration with a test problem was carried out to confirm the software functioned as expected. Since the analysis method has a general mathematical foundation, it is also applicable to other experiments, such as X-ray or electron diffraction experiments.

DOI: $10.12693 /$ APhysPolA.137.188

PACS/topics: 07.78.+s, 14.60.Cd, 68.35.Bs

\section{Introduction}

Since material properties are governed by the atomic structure or the type and position of each atom, the information of the structure is critical in discerning these properties. For bulk structure, X-ray diffraction is the standard technique used to determine the structure of the crystals of new materials, proteins, and so on. For surface structure, however, a standard technique for the definitive determination of the atomic structure of the topmost and subsurface atoms is not yet established.

TRHEPD has been developed as a novel method for such surface structure determination. At the Slow Positron Facility (SPF), Institute of Materials Structure Science (IMSS), High Energy Accelerator Research Organization (KEK) much work has been conducted, successfully revealing surface structures of the surfaces of interest (see review [1]). For example, TRHEPD determined the structures of the rutile- $\mathrm{TiO}_{2}(110)(1 \times 2)$ surface which had been the subject of debate over 30 years [2].

Here, we report recent activity on the software development for the analysis of TRHEPD data. It is based on the inverse problem in which the surface structure is determined from the experimental diffraction data.

\footnotetext{
* corresponding author; e-mail D19T1101M@edu.tottori-u.ac.jp
}

The present paper is organized as follows: experiment and theory of TRHEPD is explained briefly in Sect. 2 . Overview of the data analysis software is presented in Sect. 3. Section 4 details the technical demonstration of the software and associated discussions. A summary and a future aspect is given in Sect. 5 .

\section{TRHEPD}

Features of TRHEPD are shown schematically in Fig. 1 and will be briefly explained in this section. The experimental basis is the same as in RHEED experiments.

The problem is to determine the positions of the atoms at the top-most surface layer and several subsurface layers. Hereafter, the $z$ axis is assumed to be perpendicular to the material surface and the coordinates are denoted as $\boldsymbol{r}=(x, y, z) . N_{a}$ is the number of the atoms of which positions $\left(\left(x_{i}, y_{i}, z_{i}\right), i=1,2, \ldots, N_{a}\right)$ will be determined through the data analysis.

The data analysis provides a solution to the inverse problem in which the surface structure, $X$, is determined from the experimental diffraction data $D_{\exp }\left(D_{\exp } \Rightarrow X\right)$. The calculated diffraction data $D_{\text {cal }}$ is obtained from the position of the atoms $X[3]$. and the calculation is called the forward problem $\left(X \Rightarrow D_{\text {cal }}(X)\right)$.

\subsection{Experiment}

The TRHEPD experiment is shown schematically in Fig. 1a. The incident wave direction is characterized by the glancing angle $\theta$ and the azimuthal 


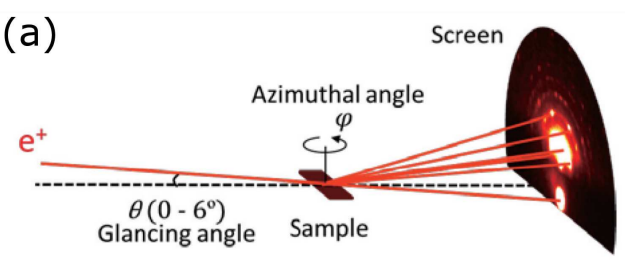

(b)

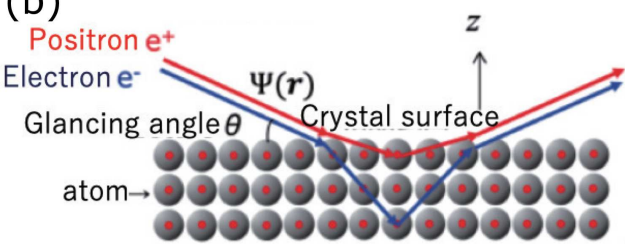

Fig. 1. Schematic diagram of (a) experimental setup and (b) typical paths of the beams for the positron (THREPD) and electron (reflection high-energy electron diffraction, RHEED) cases.

angle $\varphi$. We also define the incident wave vector $\boldsymbol{K}^{(\text {in })}=(K \cos \theta \cos \varphi, K \cos \theta \sin \varphi, 0)$ projected on the $x-y$ plane, where $K$ is the positron wave number in the vacuum.

Diffraction spots on the screen in Fig. 1a are characterized by the two-dimensional indices $(p, q)$ of reciprocal lattice rods. The intensity of the specific spots, $D_{p q}$, is observed as a function of the incident glancing angle, $\theta$ $\left(D_{p q}=D_{p q}(\theta)\right)$, and called the rocking curve. The rocking curve, $D_{p q}(\theta)$, depends also on the azimuthal angle $\varphi$, which is fixed during a rocking curve measurement. The spot with the indices of $(p, q)=(0,0)$ is usually brightest and called 00 spot. This paper focuses on the 00 spot and we will drop the indices for simplicity $(D=D(\theta))$. The observed data among discrete glancing angles is denoted as $\boldsymbol{D} \equiv\left(D\left(\theta_{1}\right), D\left(\theta_{2}\right), \ldots, D\left(\theta_{M}\right)\right)$, where a typical number of the glancing angles is $M=50-100$. The present data analysis is carried out by the normalized vector data $\boldsymbol{D}(|\boldsymbol{D}|=1)$ and similarly normalized calculated values.

It is important that the rocking curve with a given azimuthal angle $\varphi$ is hardly affected by the atomic coordinate parallel to the vector $\boldsymbol{K}^{\text {(in) }}$. If the vector $\boldsymbol{K}^{\text {(in) }}$ is parallel to the $y$-axis, for example, the rocking curve depends only on the $x$ and $z$ components of the atomic position $\left(\boldsymbol{D}=\boldsymbol{D}\left(x_{1}, x_{2}, \ldots, x_{N_{a}}, z_{1}, z_{2}, \ldots, z_{N_{a}}\right)\right)$. In addition, one can choose the azimuthal angle intentionally shifted from low-index zone axes so that the rocking curve practically depends only on the $z$ components of the atomic position $\left(\boldsymbol{D}=\boldsymbol{D}\left(z_{1}, z_{2}, \ldots, z_{N_{a}}\right)\right)$ - this is called a one-beam condition [5]. Another choice for the azimuthal angle is that it is set along a low-index zone axis - this is called a many-beam condition. These properties allow us to reduce the number of variables in the data analysis from $3 N_{a}$ to $N_{a}$. Such dimensional reduction is of great advantage in realizing fast and reliable data analysis.

Therefore, the measurement procedure, typically, consists of two stages, where three diffraction data sets at different azimuthal angles are obtained. One data set is provided by an experiment in a one-beam condition, which is denoted as $\boldsymbol{D}^{(\mathrm{OB})}$. The other two sets are provided by experiments in the many-beam condition, which are denoted as $\boldsymbol{D}^{(\mathrm{MB} 1)}$ and $\boldsymbol{D}^{(\mathrm{MB} 2)}$. Mutually orthogonal incident wave vectors $\boldsymbol{K}^{(\mathrm{in})}$ were chosen for the two data sets under the many-beam condition. The first stage of the analysis procedure determines the $z$ component of the atomic position $\left(z_{1}, z_{2}, \ldots, z_{N_{a}}\right)$ from the data set in the one-beam condition $\left(\boldsymbol{D}^{(\mathrm{OB})}\right)$. The second stage determines the two components on the $x-y$ plane, $x$ and $y$ coordinates from the other two data sets each in the many-beam condition $\left(\boldsymbol{D}^{(\mathrm{MB} 1)}, \boldsymbol{D}^{(\mathrm{MB} 2)}\right)$, in which analysis of the $\mathrm{z}$ components $\left(z_{1}, z_{2}, \ldots, z_{N_{a}}\right)$ determined in the first stage are fixed.

\subsection{Theory}

The theory or the forward problem $\left(X \Rightarrow D_{\text {cal }}(X)\right)$ of TRHEPD $[3-5]$ is based on the quantum scattering problem of the positron wave function $\Psi(\boldsymbol{r})$; the situation is shown schematically in Fig. 1b. The partial differential equation with a given glancing angle and an azimuthal angle

$$
\left(\Delta+K^{2}+U(\boldsymbol{r})\right) \Psi(\boldsymbol{r})=0
$$

is solved numerically, so as to obtain rocking curve data $\boldsymbol{D}=\boldsymbol{D}_{\text {cal }}(X)$. Here $U(\boldsymbol{r})$ is the crystal potential determined by the atomic positions $X$. The crystal potential $U(\boldsymbol{r})$ is periodic on the $x-y$ plane and can be written by the two-dimensional Fourier series

$$
U(x, y, z)=\sum_{m} U_{m}(z) \exp \left(\mathrm{i}\left(k_{x}^{(m)} x+k_{y}^{(m)} y\right)\right),
$$

where $\left(k_{x}^{(m)}, k_{y}^{(m)}\right)$ is the surface reciprocal lattice vector of the $m$-th rod $\left(p_{m}, q_{m}\right)$. In the numerical calculation, the coordinate $z$ is discretized by the mesh grid with an equi-interval $h\left(z:=z_{0}+j h, j=0,1,2, \ldots\right)$. A typical value of the mesh interval, $h$, is $h=0.02 \AA$.

The calculation under the one-beam condition [5] is much faster than that under the many-beam condition, since under the one-beam condition, only one Fourier component $\left(k_{x}^{(0)}, k_{y}^{(0)}\right)=(0,0)$ is non-zero in Eq. (2):

$$
U(x, y, z)=U_{0}(z) .
$$

In this case, the wave function can also be written as $\Psi \equiv \Psi_{0}(z)$ and Eq. (1) is reduced to a one-dimensional scattering problem

$$
\left(\frac{\mathrm{d}^{2}}{\mathrm{~d} z^{2}}+K^{2} \sin ^{2} \theta+U_{0}(z)\right) \Psi_{0}(z)=0 .
$$

This calculation method and its Fortran software was originally developed for electron diffraction (RHEED) by one of the authors (Ichimiya) [3-5] and later modifed by another (Hanada) [6]. It is applicable to TRHEPD, since TRHEPD is differs from RHEED only in the sign of the incident particle charge. As the electron beam penetrates into deeper layers than the positron, owing to refraction off the surface, as schematically shown in Fig. 1b, electron diffraction is less sensitive to surface structure than the positron diffraction. 
The forward problem solver $\left(X \Rightarrow D_{\text {cal }}(X)\right)$ software has been used in the data analysis to optimize the atomic structure $X$ [1]. Actual procedures, however, are based on trial and error without using any numerical optimization algorithm. In the present work we are developing a numerical optimization algorithm which optimizes $X$ automatically.

\section{Data analysis method}

This section details the present method used for the data analysis or the inverse problem $\left(\boldsymbol{D}_{\text {exp }} \Rightarrow X\right)$. The inverse problem is solved by optimizing the residual function between the calculated and experimental diffraction data

$$
R(X) \equiv\left|\boldsymbol{D}_{\text {cal }}(X)-\boldsymbol{D}_{\text {exp }}\right|
$$

with a given experimental diffraction data $\boldsymbol{D}_{\text {exp }}$, where $\boldsymbol{D}_{\text {cal }}$ is also normalized $\left(\left|\boldsymbol{D}_{\text {cal }}\right|=1\right)$. The function $R(X)$ is called the reliability factor or R-factor.

In general, optimization algorithms are classified into local or global searches. Two typical algorithms are illustrated in Fig. 2. Figure 2a shows a local search algorithm with iterative updates, in which an initial structure data $X^{(0)}$ is prepared and the data, $X$, is updated iteratively $\left(X^{(0)} \Rightarrow X^{(1)} \Rightarrow X^{(2)} \ldots \Rightarrow X^{(i)} \Rightarrow \ldots\right)$, so as to decrease $R(X)$. Figure $2 \mathrm{~b}$, on the other hand, shows a global search algorithm with a straightforward calculation on a mesh grid, which will be discussed later in Sect. 4.2 .
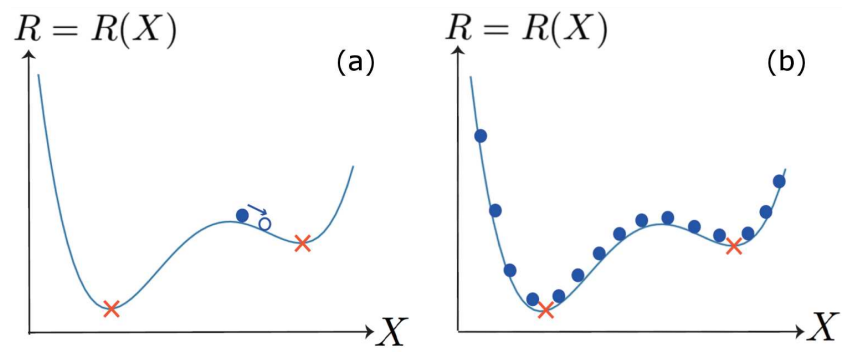

Fig. 2. Schematic figure for two optimization methods of the function $R=R(X)$ : (a) local search or the iterative optimization, (b) global search or simplecomparison optimization on grid.

The present paper focuses on a local search algorithm. The iterative optimization is realized using the NelderMead algorithm [7, 8], a commonly used gradient-free optimization algorithm, where the gradient $(\nabla R)$ is not calculated in any way. The iterative process is stopped when it reaches a convergence criteria chosen by the user. In this study, we developed a Python-based data analysis software. The Nelder-Mead algorithm is realized by the module in the scipy library (scipy.optimize.fmin). The method is standard and the use of the scipy library is not essential.

\section{Technical demonstration and discussion}

\subsection{Technical demonstration}

A numerical test problem was run for the technical demonstration of our data analysis software. The test problem used was to reproduce the $\mathrm{Si}(001)-2 \times 1$ surface structure. Studies of this structure, shown in Fig. 3, have been reported in a number of papers, such as Ref. [9]. The two top-most silicon atoms form an asymmetric dimer, where the $z$ coordinate values of these atoms differ significantly. The higher (vacuum-side) atom and the lower (bulk-side) atom of the surface dimer are denoted as $\mathrm{Si}$ (high) and $\mathrm{Si}$ (low), respectively. The asymmetry is induced to decrease the total surface energy by electron transfer from $\mathrm{Si}$ (low) to $\mathrm{Si}$ (high), which results in a lone pair in the dangling-bond state at $\mathrm{Si}($ high) and an empty dangling-bond state at $\mathrm{Si}(\mathrm{low})$. The $z$ coordinates of the higher and lower atoms are denoted by $z_{1}$ and $z_{2}$ in Fig. 3, respectively, where the origin of the $z$ axis $(z=0)$ is located at the plane of the atoms in the second surface layer.

In the present demonstration, the values of the $z$ component of the two top-most silicon atoms $\left(z_{1}, z_{2}\right)$ are determined from the diffraction data in the onebeam condition. A numerically generated "reference" data $\boldsymbol{D}_{\text {ref }}$ is used, instead of real experimental data $\boldsymbol{D}_{\text {exp }}$. The reference data $\boldsymbol{D}_{\text {ref }}$ is generated numerically for the known structure $\left(z_{1}, z_{2}\right)=\left(z_{1}^{(\text {ref })}, z_{2}^{(\text {ref })}\right)$ $\left(\boldsymbol{D}_{\text {ref }} \equiv \boldsymbol{D}_{\text {cal }}\left(z_{1}^{(\text {ref })}, z_{2}^{(\text {ref })}\right)\right)$ and the $R$-factor is defined as:

$$
R\left(z_{1}, z_{2}\right) \equiv\left|\boldsymbol{D}_{\text {cal }}\left(z_{1}, z_{2}\right)-\boldsymbol{D}_{\text {cal }}\left(z_{1}^{(\text {ref })}, z_{2}^{(\text {ref })}\right)\right|,
$$

where $\left(z_{1}^{(\text {ref })}, z_{2}^{(\text {ref })}\right)=(1.5832 \AA, 0.8603 \AA)$. A demonstration with the numerically generated reference data was carried out in order to confirm that the Nelder-Mead algorithm was suitable and reached the exact solution $\left(\left(z_{1}, z_{2}\right) \rightarrow\left(z_{1}^{(\mathrm{ref})}, z_{2}^{(\mathrm{ref})}\right)\right)$. When analyzing with experimental data, an optimized value of $R \leq 10^{-2}$ is usually acceptable in surface structure determination research.

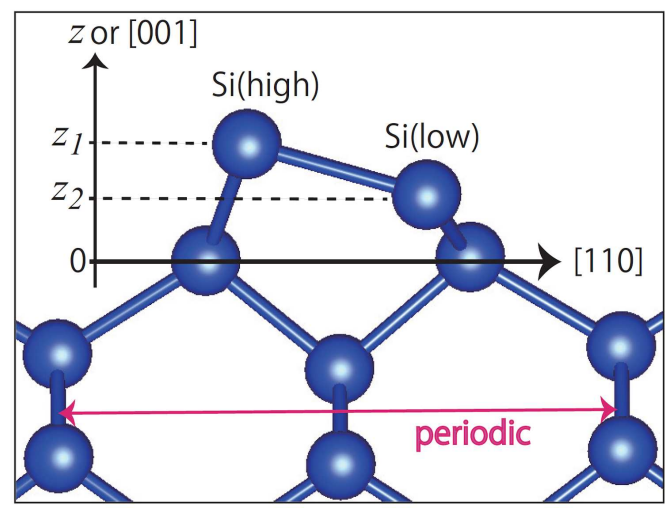

Fig. 3. Atomic positions of $\mathrm{Si}(001)-2 \times 1$ surface with an asymmetric surface dimer. The vertical axis ( $z$ axis) is along the [001] direction, while the horizontal axis is along the [110] direction. The periodic unit in the [110] direction is indicated by the red arrowed line. 

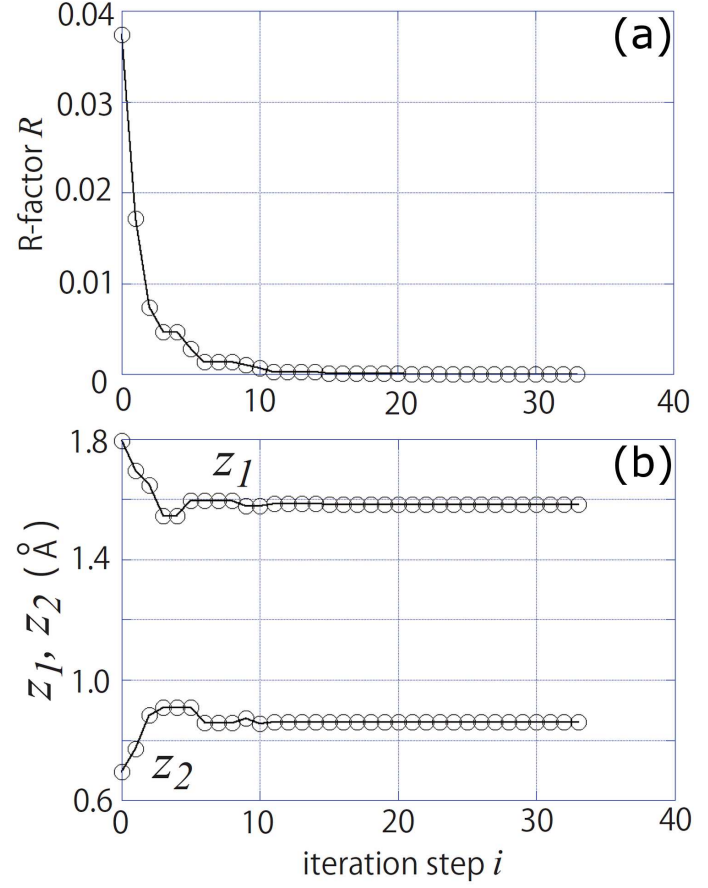

Fig. 4. The data analysis for $\operatorname{Si}(001)-2 \times 1$ surface: (a) the $R$-factor $\left(R=R\left(z_{1}, z_{2}\right)\right)$ and (b) the $z$ coordinates of the surface atoms $\left(z_{1}, z_{2}\right)$ are plotted as functions of the iteration step $i$.

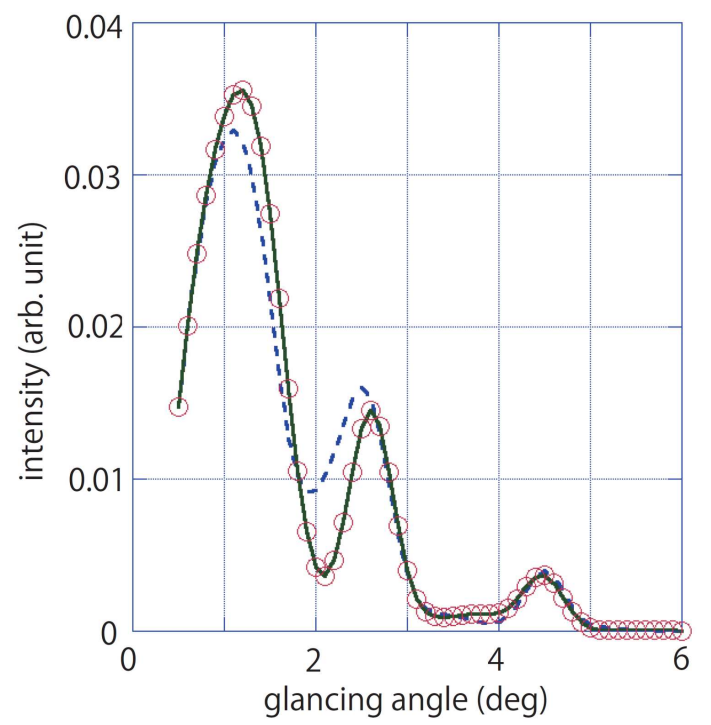

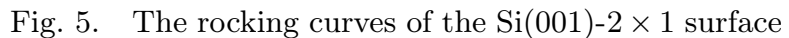
of the initial and final structures are plotted by dashed and solid lines, respectively. The rocking curve in the reference structure is also plotted by circles.

The analysis results are summarized in Figs. 4 and 5 . Figure 4 shows the iterative optimization process. The $R$-factor $\left(R=R\left(z_{1}, z_{2}\right)\right)$ and the $z$ coordinates of the surface atoms $\left(z_{1}, z_{2}\right)$ are plotted as functions of the iteration step $i$, in Fig. 4a,b, respectively. Figures 4 and 5 indicate that the structure $\left(z_{1}, z_{2}\right)$ converges to the exact solution $\left(\left(z_{1}, z_{2}\right) \rightarrow\left(z_{1}^{(\text {ref })}, z_{2}^{(\text {ref })}\right)\right)$ correctly. The $R$-factor changes from $R=0.037$ at the initial structure $(i=0)$ to $R=2 \times 10^{-6}$ at the final structure $(i=33)$. Figure 5 shows the rocking curve $D_{\text {cal }}=D_{\text {cal }}(\theta)$ in the initial and final structure, where the reference data $D_{\text {ref }}=D_{\text {ref }}(\theta)$ is also shown. Figure 5 confirms that the atomic coordinates obtained by the search reproduce the reference data correctly. The analysis was carried out on a notebook PC with an Intel Core ${ }^{\mathrm{TM}} \mathrm{i} 3-6006 \mathrm{U}$ processor and the elapsed time was $72 \mathrm{~s}$, including the computational time and the file $\mathrm{I} / \mathrm{O}$ time.

It should be noted that the difference in the atomic positions $\left(z_{1}, z_{2}\right)$ between the initial and the final structures is of the order of $0.1 \AA$ in Fig. $4 \mathrm{~b}$ and the difference can be clearly observed in the rocking curves of Fig. 5. This demonstrates that TRHEPD can give excellent selectivity or an ultrafine spatial resolution in the order of $0.1 \AA$, if the experimental uncertainty of the observed data is sufficiently small.

\subsection{Discussion}

A number of issues are discussed below on the current and prospective versions of the data analysis software. (I) The present paper has detailed a technical demonstration of the software. Data analysis with real experiment is ongoing. (II) The forward problem solver code used is planned to be parallelized for faster computation. Since computations of the intensity $\left\{D_{\text {cal }}\left(\theta_{i}\right)\right\}$ with different $i$ numbers are independent, the procedure is ideal for parallelism on current computers. (III) Work is underway to develop further software with the global search algorithm shown in Fig. 2, so as to avoid local, not absolute, optimization. The software presently used provides an iterative optimization algorithm and requires an initial guess for the structure. As such, the analysis is based on a local search algorithm and can be trapped by a local minimum of $R(X)$. A simple-comparison algorithm may require significant computational cost, because the number of sets of atomic coordinates increases exponentially with the increasing number of atoms and in reducing the mesh in the coordinates. However, this type of algorithm is suitable for modern massive parallel supercomputers as the calculations of $R=R(X)$ among different atomic positions, $X$, of different atoms are independent procedures. Our global search software is now being tested on several supercomputers, such as the OakforestPACS supercomputer in Japan. (IV) In addition, we plan to develop another global search method based on the Bayesian inference with a Monte Carlo (stochastic) algorithm. The Monte Carlo algorithm is known as a reliable and efficient sampling method. The method provides the posterior probability of atomic positions through Bayes' theorem and enables us to evaluate the uncertainty of estimated atomic positions. This method has been applied to surface structure analysis by X-ray diffraction experimentation $[10]$. 


\section{Summary and future aspect}

A data analysis software is being developed for surface structure analysis by the total-reflection high-energy positron diffraction (TRHEPD). The software provides a solution to the inverse problem, where the forward problem is a quantum scattering problem or partial differential equation. The software has a solid mathematical foundation and shows promise for the analysis of real experimental data. The program code will be available online in the near future.

As a future aspect, the software will be promoted to address general data analysis of surface structures, not only for positron diffraction but also for X-ray and electron diffraction by simply changing the forward problem solver.

\section{Acknowledgments}

The present research is supported by the Japanese government in the post-K project and KAKENHI funds (19H04125, 17H02828). Several numerical computations were carried out by the Oakforest-PACS supercomputer for the Initiative on Promotion of Supercomputing for Young or Women Researchers, Information Technology Center, The University of Tokyo, the HPCI project (hp190066) and Interdisciplinary Computational Science Program in the Center for Computational Sciences, University of Tsukuba. Several computations were carried out also on the facilities of the Supercomputer Center, the Institute for Solid State Physics, the University of Tokyo and the Academic Center for Computing and Media Studies, Kyoto University.

\section{References}

[1] Y. Fukaya, A. Kawasuso, A. Ichimiya, T. Hyodo, J. Phys. D 52, 013002 (2018).

[2] I. Mochizuki, H. Ariga, Y. Fukaya, K. Wada, M. Maekawa, A. Kawasuso, T. Shidara, K. Asakura, T. Hyodo, Phys. Chem. Chem. Phys. 18, 7085 (2016).

[3] A. Ichimiya, Jpn. J. Appl. Phys. 22, 176 (1983); ibid. 24, 1365 (1985).

[4] A. Ichimiya, S. Mizuno, Surf. Sci. 191, L765 (1987).

[5] A. Ichimiya, Surf. Sci. 191, L893 (1987).

[6] T. Hanada, H. Daimon, S. Ino, Phys. Rev. B 51 13320 (1995)

[7] A.J. Nelder, R. Mead, Comput. J. 7, 308 (1965).

[8] J.C. Lagarias, J.A. Reeds, M.H. Wright, P.E. Wright, SIAM J. Opt. 9, 112 (1998).

[9] A.A. Stekolnikov, J. Furthmüller, F. Bechstedt, Phys. Rev. B 65, 115318 (2002).

[10] M. Anada, Y. Nakanishi-Ohno, M. Okada, T. Kimura, Y. Wakabayashi, J. Appl. Crystallogr. 50, 1611 (2017). 